\title{
Factors Influencing Full Immunization Coverage among Children Aged 12-23 months in Chadereka Rural Community, Zimbabwe
}

\author{
Article by Priscilla Kusena \\ Texila American University, School of Public Health, Guyana, South America \\ E-mail: priscillamadzikus@gmail.com
}

\begin{abstract}
Full Immunization (a child having received BCG, 3 doses of oral polio, three doses of pneumococcal conjugate, three doses of pentavalent and measles vaccine) against vaccine preventable diseases reduces deaths of under-fives in developing countries. Zimbabwe records show low full immunization coverage of $69.2 \%$ in 2015 and the causalities are poorly understood. We examine factors undermining full immunization coverage among children aged 12-23 months in Chadereka community, Centenary district. We adopted a cross sectional study design using modified cluster sampling method to select study participants. Fifteen systematically selected clusters of 8 children were utilized to determine full immunization coverage. Data collection was done using a pretested structured questionnaire and analysed using excel software. Mothers $(n=120)$ with children (aged 12-23 months) were sampled and history of immunization was extracted from child's baby card plus mother's history. We found that $64.2 \%$ (95\% CI: 53.9-74.5) of study participant had achieved full immunization. Specific vaccine coverage for BCG, three doses of polio, three doses of pneumococcal conjugate, three doses of pentavalent, and measles vaccine were $90.8 \%, 89.4 \%, 86.1 \%, 82.4 \%$ and $70 \%$ respectively. Source for immunization were health centre (53.5\%), outreach services (37\%) and hospital (9.5\%). Mother's awareness (p-value 0.0317) and wealth status ( $p$ value 0.01592) were the major predictors of full immunization coverage. Reasons given for failure to fully immunize children were obstacles (74.4\%), lack of motivation (11.6\%), lack of information (9.3\%) and community factors (4.7\%). We conclude that improved awareness to mothers and empowering women potentially increase the national full immunization coverage.
\end{abstract}

Keywords: Full immunization, factors, coverage, vaccine preventable diseases.

\section{Introduction}

Immunizing children reduces deaths of under-fives in developing countries ${ }^{30}$. It is estimated that every year 27 million children worldwide do not receive vaccination ${ }^{31}$. In addition, 2 million children are estimated to die of vaccine preventable diseases globally ${ }^{31}$. In 2013, 145,700 of the child deaths were due to measles ${ }^{34}$. In low and middle income countries, immunization is effective in protecting children against vaccine preventable diseases ${ }^{19}$. Vaccination to children prevent 2.5 million deaths per year ${ }^{30}$.

The World Health Organization (WHO) introduced the Expanded Programme on Immunization (EPI) to control vaccine preventable diseases among children in $1974^{17}$. Zimbabwe launched Expanded Program on Immunization in 1982 to reduce child morbidity and mortality from six vaccine preventable diseases. Child vaccination services are offered at most health facilities in Zimbabwe by qualified health personnel. However, full vaccination coverage remains low compared to the required World Health Organization target of $90 \%$ at national level. The national level full immunization coverage is $69 \%{ }^{24}$. Mashonaland Central Province has a full immunization coverage of $67 \%$. This is despite that routine immunization is offered at most health facilities in the province.

Several studies show that full immunization coverage is hampered by long distance of travel to health facility ${ }^{6}$, missed opportunity ${ }^{1}$, poor socioeconomic status ${ }^{18}$, high birth $\operatorname{order}^{25}$ and low educational status of mother ${ }^{14}$. Studies done in Zimbabwe show the effect of poor logistical arrangements and health systems on 
DOI: $10.21522 /$ TIJPH.2013.05.04.Art011

ISSN: $2520-3134$

vaccination of children ${ }^{3,23}$. The Ministry of Health and Child Care view that high attrition of experienced and skilled personnel resulted in low immunization at health facilities as there was lack of staff offering vaccination services ${ }^{21}$.

At Chadereka health facility, the dropout rate for measles vaccine compared to pentavalent one ranges from $14 \%$ to $52 \%$ for the period January-August $2015^{12}$. This is despite that vaccination services are routine. In addition, outreach services are offered at four villages in the catchment area of the health facility monthly. Furthermore, the health facility utilizes the supermarket approach to offer vaccination services to clients in their catchment area $^{21}$. Monthly, the district Community Nursing Department offers supportive supervision to health facility staff and mentors them on vaccination.

The study will determine full immunization coverage for Chadereka health facility and elicit the reasons for immunization failure to inform program authorities. Identifying factors influencing full immunization coverage will assist in determining the most critical factors that affect full immunization status in Chadereka Community. There is little information about factors affecting full vaccine uptake at Chadereka health facility although vaccination coverage for specific antigens are available in the district. Results from the study will contribute towards establishing the full immunization coverage and will be used by National, Provincial and EPI focal persons to improve full vaccination uptake within districts.

\section{Methods}

\section{Description of study site}

The study was conducted in Centenary District of Mashonaland Central Province in Zimbabwe. The district was reported to have low childhood immunization status in the province (District Health Information Report, 2015). Chadereka health facility is a council owned health facility with a catchment population of 23,054. The under-one population which was expected to be vaccinated in 2013 and 2014 was 816 and 830 respectively.

\section{Study population}

The study population comprised of 120 children aged 12-23 months of age. Modified cluster sampling method was applied to select eligible children and give information on immunization coverage ${ }^{8}$. Fifteen clusters and eight children from each cluster were chosen because the sample size gives an accuracy of 10 $\%$ and $95 \%$ Confidence interval ${ }^{1}$. Level of accuracy is based on the assumption of high level of difference in coverage between clusters ${ }^{1}$.

\section{Study duration}

The study was conducted from September 2015 to December 2015. Pilot study was conducted in another district for validation of data collection tools in November 2015. Questions were adjusted accordingly after the pretesting of the questionnaire.

\section{Sampling method}

Chadereka health facility has 43 villages. Modified cluster sampling technique was used to select respondents. Fifteen systematically selected clusters of 8 children was utilized to determine full immunization coverage at Chadereka health facility. Each of the 15 clusters comprised of 8 children in the 12-23 months age group. Selection of the clusters was done by listing down all the villages in Chadereka catchment area with corresponding population. Cumulative population was done per village by adding the previous total population to the population for the next village. Sampling interval was calculated and a random number was selected using a currency note. The random number had the same number of digits as the number of digits on sampling interval. The random number identified was less than the sampling interval. First cluster was identified by locating the first community listed which had the cumulative population equals or exceeding the random number. To identify the next village, the random number was added to the sampling interval. Again the cumulative population for cluster 2 was greater than or equal to 
the total of random number and sampling interval. See attached Annex a on cluster identification in this study. The next cluster was identified through adding the sampling interval to the current cumulative population of the next village.

Selection of households in identified clusters was done randomly. A list of households in identified clusters of Chadereka community was obtained from the health facility Environmental and Health Department. Households were numbered from 1 to the highest number. A random number was selected using a currency note from one to the highest number on the household list (inclusively). The household (HH) whose number corresponded to the random number selected was the first HH visited. The nearest subsequent household whose front door was next to the first was selected as the second household until eight children were selected. If there was more than one child in the same family eligible for study, the youngest child was chosen.

\section{Inclusion and exclusion criteria for study population}

Study population included children born exactly 12 months from the date the interviews were conducted and any age before 24 months. If there are two young children from the same family eligible for the study, the youngest child was selected to participate in the study. Children who were visitors were excluded in the study. In addition, children whose parents refused to participate in the study were excluded as well as those with parents who had communication challenges. The child's care giver was defined as any mother, father or anybody attending to the needs of the child.

\section{Sample size}

Sample size was calculated using the World Health Organization (WHO) modified cluster sampling method $^{8}$. The expected full immunization coverage for the district is $85 \%$, precision of $=+/-10 \%(d=10)$, design effect of $2(\mathrm{DE}=2), 95 \%$ confidence interval $^{1}$

$$
n_{\text {min }}=\mathrm{DE} \times \frac{Z^{2}}{\mathrm{~d}^{2}} \frac{x p \times(1-p)}{(1-\alpha)}
$$

Therefore sample size equals:

$$
\begin{aligned}
& n=2 \times \frac{1.96^{2} \times 0.85 \times(1-0.85)=97.96}{10^{2}} \\
& \mathrm{n}=98
\end{aligned}
$$

- Being a district with poor road network, we expected a non-response rate of $20 \%$, taking the nonresponse rate thus by dividing by 0.8 sample size of 123 was obtained.

- A sample with 15 clusters was chosen in this study. Therefore per cluster: $123 / 15=8.2$ children per cluster, rounded off to 8 children per cluster.

- The total sample size was 15 clusters multiplied by 8 children per cluster to give 120 children

\section{Study tools}

Structured questionnaires were used to collect data on factors influencing full immunization coverage among children aged 12-23 months. Pretesting was done in another district in Mashonaland Central Province. Ambiguous questions were rephrased following pretesting.

\section{Collection of data}

Data collection was done using a structured questionnaire to identify factors associated with full immunization coverage and determine the full immunization coverage at Chadereka health facility. The structured questionnaire was administered by the researcher and five nurses trained by the researcher. The study interviewed the child's mother, and for those who were not raised by their mother, the person taking care of the child was interviewed. Questionnaires covered information on physical access, health worker knowledge, attitudes and practices, missed opportunity, caregiver factors, community and societal factors and vaccine availability and child vaccination status.

Full immunization status was determined by asking the caregiver to produce a vaccination card for the 
DOI: $10.21522 /$ TIJPH.2013.05.04.Art011

ISSN: $2520-3134$

child and also to give history of vaccination of their child in cases where the card had been lost. WHO manual on EPI coverage describes two alternatives for estimating uptake of vaccines, one is by "card only ${ }^{40}$. Vaccinations have to be documented on the card, with the date of vaccination on the numerator and batch number on the denominator. The second one is by 'card plus history', which included in numerator all reported vaccination including those not documented other than the mother's report.

Questionnaire on reason for failure to immunize was administered to caregivers whose children were partially or not immunized. Respondents were asked to state the single most important reason why the child was not fully immunized. In cases where the response by the caregiver is not among options given in the questionnaire, interviewers would capture verbatim responses for analysis later with other team members.

\section{Data analysis}

Collected data was entered, coded and analysed using Excel software. The frequency of continuous and categorical variables, means and standard deviation of age were calculated. Chi-square test was used to test statistical associations and p-value at $5 \%$ significance level.

\section{Ethical consideration}

Permission to conduct the study was granted by the District Health Executive together with Catholic Organization for Relief and Development Aid Country Office. This was a district generated request in making effort to understand immunization coverage at the health facility. Caregivers were given informed consent and signed to agree participation in this study.

\section{Results}

This chapter presents findings of the study under selected characteristics of mother and child in relation to full immunization at Chadereka health facility. In addition, reasons for failure to immunize children are also presented in this chapter.

\section{Sociodemographic characteristics of study population}

A total of 120 mothers of children aged 12-23 months were selected for interviews and the response rate was $100 \%$. Majority of the children were females (53.3\%). A total of $78(65 \%)$ children were aged between 14 and 20 months and the mean age was 17.8 months (SD 3.4). All respondents were mothers of the children targeted in the study. The mean age of the mothers was $27.5(\mathrm{SD}=7)$ years, which ranged from 15 to 56 years. Table 4.1 below presents the socio-demographic characteristics of mothers and children.

Only $45 \%$ of the mothers had secondary education compared to $55 \%$ who had primary education. Majority of them (64.2\%) were not aware of the immunization services offered at their health facility. Minority (32.5\%) of the mothers were of medium wealth status whereas the majority were poor $(67.5 \%)$. Majority (94.2\%) were married at the time of the study and few (5.8\%) were divorced. Mothers were mainly of the apostolic sect (79.2\%) whilst 8.3\% did not report any religion. 9.2\% mothers had a birth order of 6 children or more. Majority of the mothers (47.5\%) had a birth order of 2 to 3. 
Texila International Journal of Public Health Volume 5, Issue 4, Dec 2017

Table 4.1. Sociodemographic characteristics of mothers and child at chadereka health facility

\begin{tabular}{|c|c|c|}
\hline Characteristic & Frequency & Percent \\
\hline \multicolumn{3}{|l|}{ Sex of the child } \\
\hline Male & 56 & 46.7 \\
\hline Female & 64 & 53.3 \\
\hline \multicolumn{3}{|c|}{ Age group in months } \\
\hline 12 to 13 & 13 & 10.8 \\
\hline $14-23$ & 107 & 89.2 \\
\hline \multicolumn{3}{|l|}{ Mothers } \\
\hline $15-24$ & 44 & 36.7 \\
\hline $25-34$ & 54 & 45.0 \\
\hline $35+$ & 22 & 18.3 \\
\hline \multicolumn{3}{|l|}{ Educational Status } \\
\hline Primary & 66 & 55.0 \\
\hline Secondary & 54 & 45.0 \\
\hline University & 0 & 0.0 \\
\hline \multicolumn{3}{|c|}{ Mother's Awareness } \\
\hline Not Aware & 77 & 64.2 \\
\hline Aware & 43 & 35.8 \\
\hline \multicolumn{3}{|l|}{ Wealth Status } \\
\hline Poor & 81 & 67.5 \\
\hline Medium & 39 & 32.5 \\
\hline Rich & 0 & 0.0 \\
\hline \multicolumn{3}{|l|}{ Marital Status } \\
\hline Never Married & 0 & 0.0 \\
\hline Currently Married & 111 & 92.5 \\
\hline Divorced & 9 & 7.5 \\
\hline Widowed & 0 & 0.0 \\
\hline \multicolumn{3}{|l|}{ Religion } \\
\hline Christian & 15 & 12.5 \\
\hline Apostolic & 95 & 79.2 \\
\hline Jewish & 0 & 0.0 \\
\hline Other & 10 & 8.3 \\
\hline \multicolumn{3}{|l|}{ Birth Order } \\
\hline 1 & 23 & 19.2 \\
\hline 2 to 3 & 57 & 47.5 \\
\hline 4 to 5 & 29 & 24.2 \\
\hline $6+$ & 11 & 9.2 \\
\hline Total & 120 & 100 \\
\hline
\end{tabular}


DOI: $10.21522 /$ TIJPH.2013.05.04.Art011

ISSN: $2520-3134$

\section{Immunization coverage by card plus history}

Based on the vaccination card plus history, $64.2 \%$ (95\% CI: 53.9-74.5) were fully immunized, 10 (8.3\%, 95\% CI: 4.3-12.3) of the children were not immunized and 27.5\% (95\% CI: 17.1-37.9) were partially immunized (Table 5). The most frequently received vaccine was OPV. Particularly for OPV1, 93.3\% of the children were vaccinated. This was followed by BCG with $90.8 \%$ and Pentavalent 1 (90.8\%). OPV2 was taken by $90 \%$ of the children and Pentavalent 2 was taken by $85.8 \%$ of the children. The least taken vaccines were Pentavalent 3 with $82.5 \%$ and measles with $70 \%$. Results from the study show a decrement from first dose vaccine to the last dose (Table 4.2)

Table 4.2. Immunization coverage by card and card plus mother's history, Chadereka health facility

\begin{tabular}{|c|c|c|l|l|}
\hline & \multicolumn{2}{|c|}{ Card plus history } & \multicolumn{2}{l|}{ Card only } \\
\hline Vaccine & Frequency & Percent & Frequency & Percent \\
\hline BCG & 109 & 90.8 & 95 & 79.2 \\
\hline OPV1 & 112 & 93.3 & 95 & 79.2 \\
\hline OPV2 & 108 & 90.0 & 89 & 74.2 \\
\hline OPV3 & 102 & 85.0 & 90 & 75.0 \\
\hline Pentavalent & 109 & 90.8 & 90 & 75.0 \\
\hline $\begin{array}{c}\text { Pentavalent } \\
2\end{array}$ & 103 & 85.8 & 91 & 75.8 \\
\hline $\begin{array}{c}\text { Pentavalent } \\
3\end{array}$ & 99 & 82.5 & 87 & 72.5 \\
\hline $\begin{array}{c}\text { Measles } \\
\text { Not } \\
\text { immunized }\end{array}$ & 84 & 70.0 & 74 & 61.7 \\
\hline $\begin{array}{c}\text { Partially } \\
\text { immunized }\end{array}$ & 33 & 8.3 & 10 & 8.3 \\
\hline $\begin{array}{c}\text { Fully } \\
\text { immunized } \\
\text { 12-23 months }\end{array}$ & 77 & 27.5 & 20 & 16.7 \\
\hline $\begin{array}{c}\text { *Percentages } \\
\text { added to hundred. }\end{array}$ & cannot be & 64.2 & 65 & 54.2 \\
\hline
\end{tabular}

\section{Immunization coverage by card only}

Of the total surveyed children aged 12-23 months, vaccination card was seen and confirmed for 95 (79.2\%) children. 79.2\% took BCG and OPV1 vaccines. This was followed by Pentavalent $3(75.8 \%)$ and Pentavalent 1 and 2 (75\%). Measles vaccine was administered to $61.7 \%$ children. Of the $54.2 \%$ that were fully immunized by card only, only $38.3 \%$ were fully immunized below 1 year of age by card only. The coverage for individual vaccines by card were lower than the coverage's for card and history (Table 4.2). 


\section{Immunization by source}

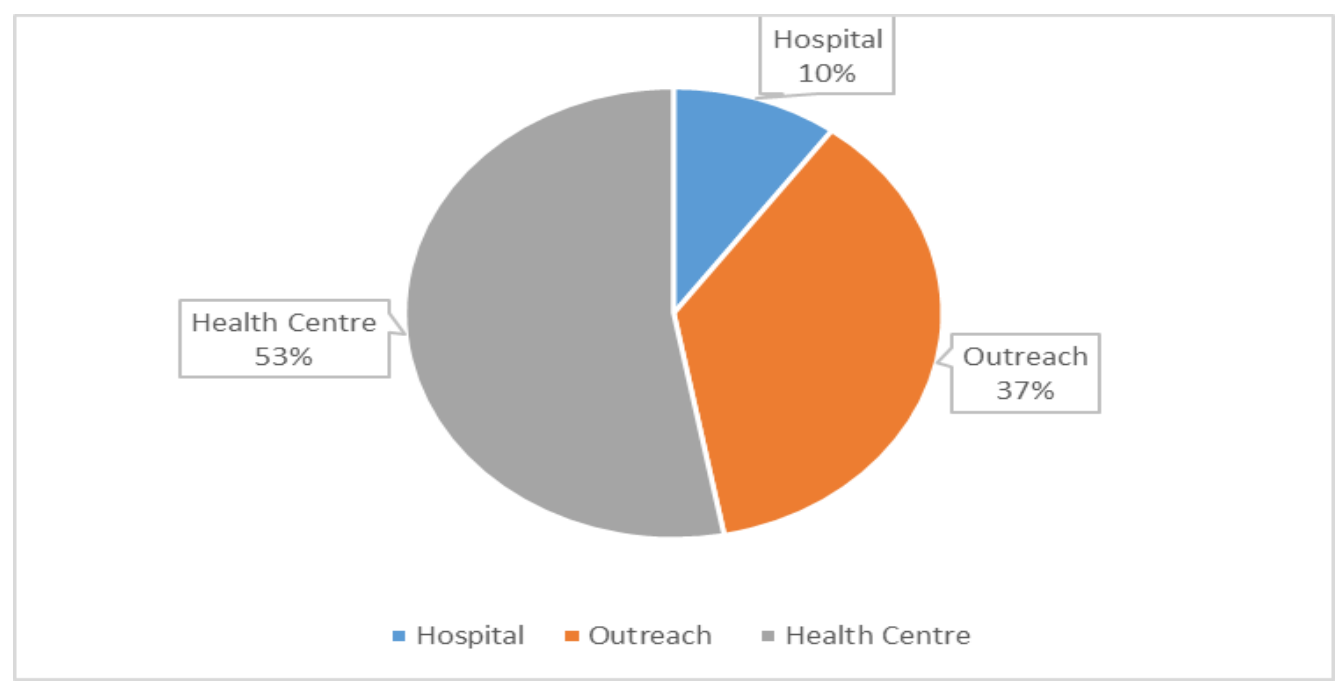

Figure 4.1. Immunization of surveyed children by source

The most frequently used source for immunization was the health center with $53.5 \%$ vaccine doses being administered there (Figure 4.1). This was followed by outreach services which contributed 37\% of the vaccination doses administered to children. The least used source for vaccination was the hospital (9.6\%).

\section{Reasons for immunization failure}

In total, failure to immunize were mainly a result of obstacles (74\%) followed by lack of motivation (12\%). Lack of information contributed to failure to immunize by (9\%).Community factors $(5 \%)$ were the least cited as reason for immunization failure (Figure 4.2).

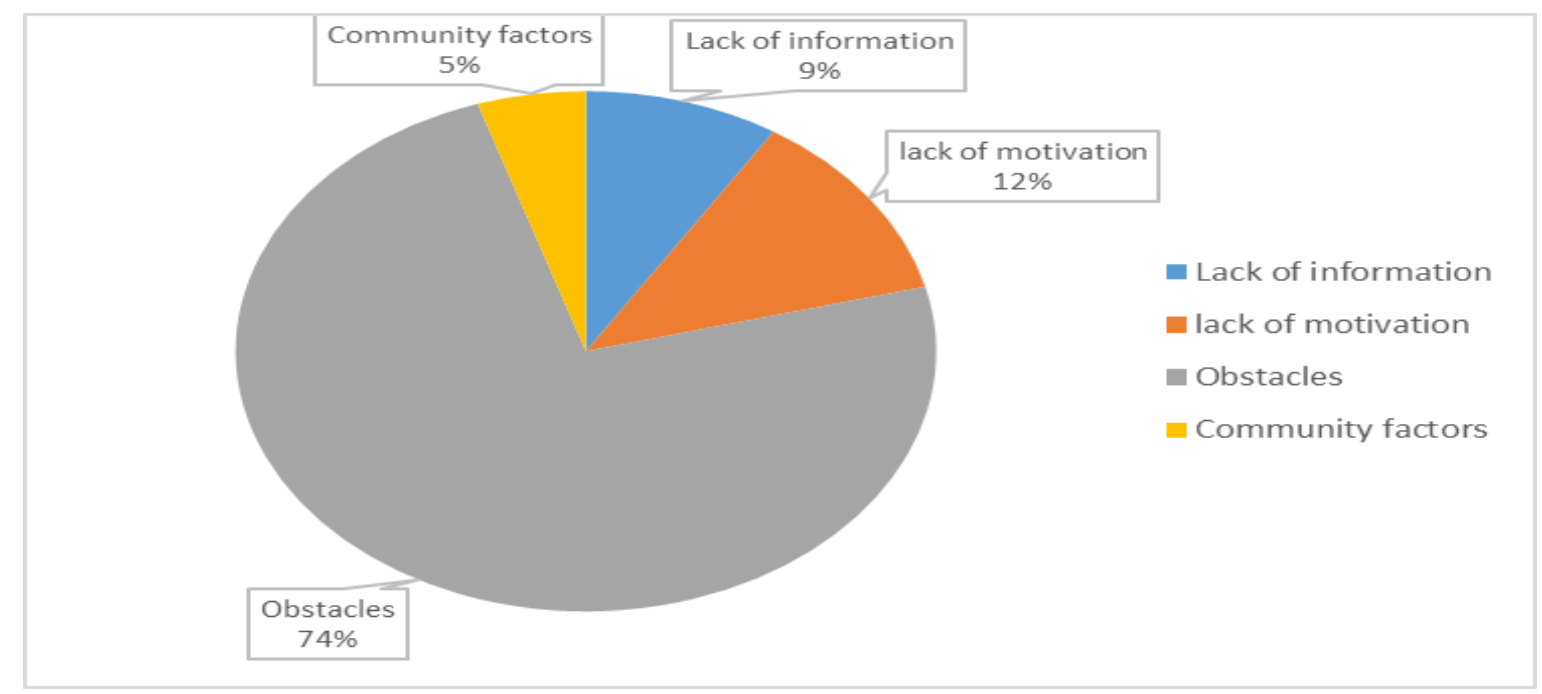

Figure 4.3. Major reasons for immunization failure

Table 4.3 below shows reasons for immunization failure reported by forty three participants from the study. Participants mentioned that lack of information on the place of time for immunization (6.98\%) and that they were unaware of need for immunization $(2.3 \%)$ as reasons for failure to immunize. Some participants cited lack of motivation to immunize children and postponed until another time (6.98\%). Sixteen percent reported that the place of immunization was too far and also $16.3 \%$ reported that the child was ill and not brought for immunization. Whilst $13.95 \%$ reported that the mother was too busy to take the 
DOI: $10.21522 /$ TIJPH.2013.05.04.Art011

ISSN: $2520-3134$

child for immunization. Religious reasons were mentioned by $4.7 \%$ as reason for failure to immunize children.

Table 4.3. Reasons for immunization failure

\begin{tabular}{|c|c|c|}
\hline \multicolumn{2}{|c|}{ Reason for failure to immunize (Partially and not immunized: $\mathrm{N}=43$ ) } & Total, n (\%) \\
\hline \multirow{5}{*}{ Lack of information } & Unaware of need for immunization & $1(2.3 \%)$ \\
\hline & $\begin{array}{l}\text { Unaware of need to return for } 2 \text { nd and 3rd } \\
\text { dose }\end{array}$ & $x_{2}=1$ \\
\hline & Place of time of immunization unknown & $3(6.98 \%)$ \\
\hline & Fear of side reactions & - \\
\hline & Wrong ideas about contraindications & - \\
\hline \multirow{3}{*}{ Lack of motivation } & Postponed until other time & $3(6.98 \%)$ \\
\hline & No faith in immunization & $2(4.7 \%)$ \\
\hline & Rumors & - \\
\hline \multirow{11}{*}{ Obstacles } & Place of immunization too far & $7(16.3 \%)$ \\
\hline & Time of immunization inconvenient & $1(2.3 \%)$ \\
\hline & Vaccinator absent & $2(4.7 \%)$ \\
\hline & Vaccine not available & $3(6.98 \%)$ \\
\hline & Mother too busy & $6(13.95 \%)$ \\
\hline & Family problem & $4(9.3 \%)$ \\
\hline & Child ill not brought & $7(16.3 \%)$ \\
\hline & Child ill brought but not immunized & - \\
\hline & Long waiting time & - \\
\hline & Geographic barrier mobility & $2(4.7 \%)$ \\
\hline & Seasonal barrier to mobility & - \\
\hline \multirow{4}{*}{ Community factors } & Religious reasons & $2(4.7 \%)$ \\
\hline & Scientific reasons & - \\
\hline & Cultural reasons & - \\
\hline & Total & - \\
\hline
\end{tabular}

\section{Factors influencing full immunization coverage}

Table 4.4 shows bivariate analysis of sociodemographic characteristics of mothers influencing full immunization coverage among 12-23 months children of age at Chadereka health facility. Chi-square test was done for each variable to determine the effect of independent variables on the outcome variable. Bivariate analysis showed that two factors (Mothers awareness: p-value 0.0317, $\mathrm{X}^{2} \mathrm{Test}=4.6105$, Wealth status: $\mathrm{p}$ value $0.01592, \mathrm{X}^{2}$ Test $=5.8981$ ) statistically significant with $\mathrm{p}$ values less than 0.05 . 
Table 4.4. Bivariate analysis of factors influencing full immunization at Chadereka health facility, November 2015

\begin{tabular}{|c|c|c|c|c|}
\hline Factors & $\begin{array}{l}\text { Partially and } \\
\text { unimmunized (n, \%) }\end{array}$ & $\begin{array}{l}\text { Fully } \\
\text { immunized (n, } \\
\%)\end{array}$ & $\begin{array}{l}\text { Chi- } \\
\text { square X2 }\end{array}$ & $P$ value \\
\hline \multicolumn{5}{|l|}{ Mothers age } \\
\hline $15-24$ & $14(11.7)$ & $30(25)$ & \multirow[t]{3}{*}{2.0827} & \multirow[t]{3}{*}{0.3529} \\
\hline $24-34$ & $23(19.2)$ & $31(25.8)$ & & \\
\hline $35+$ & $6(5)$ & $16(13.3)$ & & \\
\hline \multicolumn{5}{|l|}{ Education level } \\
\hline Primary & $23(19.2)$ & $43(35.8)$ & \multirow[t]{2}{*}{0.061869} & \multirow[t]{2}{*}{0.8496} \\
\hline Secondary & $20(16.7)$ & $34(28.3)$ & & \\
\hline \multicolumn{5}{|c|}{ Mothers awareness } \\
\hline Not aware & $10(8.3)$ & $33(27.5)$ & \multirow[t]{2}{*}{4.6105} & \multirow{2}{*}{$\begin{array}{l}0.031776 \\
\text { OR=2.5 }\end{array}$} \\
\hline Aware & $33(27.5)$ & $44(36.7)$ & & \\
\hline \multicolumn{5}{|l|}{ Wealth status } \\
\hline Poor & $35(29.2)$ & $46(38.3)$ & \multirow[t]{2}{*}{5.8981} & \multirow{2}{*}{$\begin{array}{l}0.015157 \\
\mathbf{O R}=\mathbf{0 . 3}\end{array}$} \\
\hline Medium & $8(6.7)$ & $31(25.8)$ & & \\
\hline \multicolumn{5}{|l|}{ Marital status } \\
\hline Currently married & $40(33.3)$ & $71(59.2)$ & \multirow[t]{2}{*}{1.3533} & \multirow[t]{2}{*}{0.2929} \\
\hline Divorced & $5(4.2)$ & $4(3.3)$ & & \\
\hline \multicolumn{5}{|l|}{ Religion } \\
\hline None & $5(4.2)$ & $5(4.2)$ & \multirow[t]{3}{*}{2.5504} & \multirow[t]{3}{*}{0.2794} \\
\hline Apostolic & $35(29.2)$ & $60(50)$ & & \\
\hline Christian & $3(2.5)$ & $12(10)$ & & \\
\hline \multicolumn{5}{|l|}{ Birth order } \\
\hline 1 & $7(5.8)$ & $16(13.3)$ & \multirow[t]{4}{*}{2.1771} & \multirow[t]{4}{*}{0.5365} \\
\hline 2 to 3 & $19(15.8)$ & $38(31.7)$ & & \\
\hline 4 to 5 & $11(9.2)$ & $18(15)$ & & \\
\hline $6+$ & $6(5)$ & $5(4.2)$ & & \\
\hline
\end{tabular}

\section{Limitations of study}

The study was done in Chadereka health facility catchment population. Generalization of findings to other health facility catchment population might not be possible since characteristics of caregivers may not be the same. However, the sample size was calculated by proven scientific methods and hence results could be utilized for planning purposes at the health facility.

\section{Discussion}

Coverage for full immunization by card plus history in this study (64.2\%) was lower than the national full immunization coverage $(69 \%)^{13}$ and provincial coverage $(67 \%)^{33}$. The low coverage's elicited in this study show that the health facility immunization coverage is performing lower than both the national and provincial rates. Hence the need for strengthening vaccination services at both health center and outreach services.

Mother's awareness was significantly associated with full immunization coverage among children in the study ${ }^{13,4,36}$. Mothers who were aware of immunization services offered at health facility were more likely to fully immunize their children (36.7\%) compared to their counterparts $27.5 \%$ who were not aware. In this 
DOI: $10.21522 /$ TIJPH.2013.05.04.Art011

ISSN: $2520-3134$

study, majority of the respondents $(64.2 \%)$ were not aware of immunization service offered at the health facility. Awareness of the mother on vaccination services encourages the mother to seek for the health service $^{13}$. Hence awareness plays a key role in improving vaccination coverage's.

The study found out that wealth status was also significantly associated with full immunization coverage $^{18,15}$. Mothers who were of medium wealth status were most likely to immunize their children compared to mothers who had a poor wealth status ${ }^{15}$. Less than $10 \%$ of the children from families with medium wealth status were partially immunized compared to $29.2 \%$ from poor families. The majority of mothers in the study were poor $(67.5 \%)$ and hence their children less likely to be fully immunized. Wealth status and mothers awareness were the independent determinants of full immunization in Chadereka community.

Findings from the study indicate that there are high coverage's for vaccines at birth compared to other vaccines over time (BCG coverage $90.8 \%$ compared to measles which is $70 \%)^{26}$. High coverage for BCG indicates that the community is accessing the health facility for vaccination services at the initial stages when the child is born. However, the decrement in relative vaccines indicates that there is poor continuity of vaccination to children ${ }^{30}$. This is also evidenced by the coverage for measles $(70 \%)$.

There were higher coverage's for immunization by card plus history compared to card only. Recall biases by mothers of vaccination done may result in overestimation of the coverage rates. However, the study also determined full immunization coverage by card only. Children who were partially immunized by card plus recall were $27.5 \%$ compared to those by card only (20\%). It is crucial for children to be fully immunized because incomplete vaccinations result in high infant mortality rates and also in the occurrence of childhood vaccine preventable diseases.

The study found out that the most common used source for immunizing children was the health center (53\%) followed by outreach services (37\%). Findings from the study confirm that outreach services are crucial in complementing vaccination services offered at the health center and in the community. Furthermore, majority (16.3\%) of the respondents who reported that obstacles were a major reason for failure to immunize mentioned the place of immunization being too far $^{7,6}$. Hence the need to strengthen outreach service provision for immunizations. Respondents cited various obstacles as reasons for immunization failure. Respondents pointed out that mothers are too busy to take the children for immunization $^{28}$. This concurs with findings from other studies ${ }^{20,6}$.

The place of time for immunization was unknown by respondents and in some cases the vaccine and vaccinator were absent. Findings from the study were confirmed by other studies ${ }^{33},{ }^{23}$ Child illness contributed to failure for immunization since caregivers did not bring child for immunization. This agrees with findings from other studies ${ }^{3,23}$.

\section{Conclusion}

In conclusion, maternal awareness of vaccination services offered at health facility and maternal wealth status significantly influence full immunization among children aged 12-23 months in Chadereka community. Majority of the mothers were not aware of the immunization services offered at their health facility. This might affect seeking of immunization services by the community.

Findings from the study indicate high coverage's for vaccines at birth followed by low coverage's for vaccines administered later. This decrement of vaccine coverage is worrisome since it indicates poor utilization of vaccination services.

There is low full immunization coverage for children under one year of age. Hence timely vaccinations are still a challenge in this community. Also, there is high dropout rate for measles relative to vaccines administered at birth. This indicates poor continuity of vaccination services at the health facility.

Reasons for failure to immunize were mainly due to obstacles encountered by mothers such as health facility being too far to walk and that mothers were too busy to go health facility for vaccination of their children. Child's illness was also cited as reason for immunization failure. 
Outreach services were utilized by the community to access vaccination services. Findings confirm the notion that outreach services indeed compliments health facility activities in improving vaccination coverage's. This is crucial in reaching clients that are hard to reach in the community.

Findings from this study are crucial in designing strategic solutions towards the achievement of the district full immunization target of $85 \%$. Hence, target achievement ensures achievement of the Millennium Development Goal 4.

\section{References}

[1].Abdulraheem I. S.1*, O. A. (2011). Reasons for incomplete vaccination and factors for missed opportunities among rural Nigerian children. Journal of Public Health and Epidemiology Vol. 3(4), pp. 194-203, April 2011, 194203.

[2].Aboubakary Sanou1, 2. S. (2009). Assessment of factors associated with complete immunization coverage in children aged 12-23 months: a cross-sectional study in Nouna district, Burkina Faso. BMC International Health and Human Rights.

[3].Addmore Chadambuka, A. C. (MAY 2011). The need for innovative strategies to improve immunization services in rural Zimbabwee. Impact Factor: 0.69 - DOI: 10.1111/j.1467-7717.2011.01246.x · Source: PubMed, 162-172.

[4].Akinola Ayoola Fatiregun, A. O. (2012). Maternal Determinants of complete child immunization among children aged 12-23 months in a southern district of Nigeria. Vaccine 30, 730-736.

[5].Andersen RM, Newman JF. Societal and Individual Determinants of Medical Care Utilization in the United States." Milbank Quarterly. 1973; 51(1):95-124. [PubMed]

[6].Bakirci, S. D. (2006). Vaccination coverage and reasons for non-vaccination in district of Istanbul. BMC Public Health, 125.

[7].BE Owumi, J. A. (2013). Factors Influencing Infant Immunization Uptake in the Yoruba Community of Southwestern Nigeri. Community Medicine \& Health Education, volume 3: Issue 4.

[8].Belachew Etana1, 2W.D. (2012). Factors associated with complete immunization coverage in children aged 1223 months in Ambo Woreda, Central Ethiopia. BMC Public Health 2012, 12: 566

[9].Bhola Nath, J. V. (2007). A study on determinants of immunization coverage among 12-23 months old children in urban slums of Lucknow district, India. Indian Journal of Medical Sciences, 598-606.

[10]. Dutton D.B. 1978.Explaining the Low Use of Health Services by the Poor: Costs, Attitudes or Delivery System. American Sociological Review 43 (3):348-68.

[11]. Fu-Qiang Cui, R. G. (2007). Immunization coverage and its determinants in children aged 12-23 months in Gansu, China. Elsevier, 664-671.

[12]. Health Facility Report. (2015). Vaccination Targets

[13]. Henok Tadesse, A. D. (2012). Explorative assessment of factors affecting child immunization in Wonago district, Gedeo zone, South Ethiopia. Archives of Medical Sciences, 233-240.

[14]. Jani Jagrati V1. 2C De. S. (2008) Risk factors for incomplete vaccination and missed opportunity for immunization in rural Mozambique. BMC Public Health 2008, 8: 161

[15]. Juliet N Babirye1, 2. E. (2011). More support for mothers: a qualitative study on factors affecting immunization behavior in Kampala, Uganda. BMC Public Health 2011, 11:723, 11: 723.

[16]. Kambarami R.A1, N.K.J2 (1991). Measles Epidemic in Harare, Zimbabwe despite high immunization coverage rates. Bulletin of the World Health Organization, 69 (2): 213-219

[17]. Keja K1.2C.C. (1988). Expanded programme on immunization. World Health Stat Q. 1988;41(2):59-63

[18]. Kusuma Y.S1. 2K. R. Migration and immunization: determinants of childhood immunization uptake among socioeconomically disadvantaged migrants in Delhi, India. Trop Med International Health. 2010 Nov;15(11):132632

[19]. Lee S. (2005). Demand for immunization, parental selection, and child survival: Evidence from rural India. Review of Economics of the Household 3:171-197

[20]. Lilian Chepkemoi Maina1, \&. S. (2013). Immunization coverage and its determinants among children aged 12 23 months in a peri-urban area of Kenya. Pan African Medical Journal. 
DOI: $10.21522 /$ TIJPH.2013.05.04.Art011

ISSN: $2520-3134$

[21]. Ministry of Health and Child Care. (2015).

[22]. Mohitul Ameen Ahmed Mustafi1, D. M. (2013). Factors influencing of child immunization in Bangladesh. International Journal of Mathematics and Statistics Studies, 55-65.

[23]. Muchekeza M, C. A et al. Adverse Events Following Immunization (AEFI) Surveillance in Kwekwe District, Midlands Province, Zimbabwe, 2009-2010. J Vaccines Vaccine. 2014;5:2

[24]. Multiple Indicator Cluster Survey. (2014). Key Findings Report

[25]. Mutua M. K. (2015). Determinants of immunization inequality among urban poor children: evidence from Nairobi's informal settlements. International Journal for Equity in Health 2015, 14: 24

[26]. Olumuyiwa O Odusanya, E. F. (2006). Determinants of vaccine coverage in rural Nigeria. BMC Public Health.

[27]. Saheed Gigado1, P.N. (2014). Determinants of routine immunization coverage in Bungudu, Zamfara State, Northern Nigeria. Pan African Medical Journal, 2014:18

[28]. Sarah Payne, J. T. (2013). Achieving comprehensive childhood immunization: an analysis of obstacles in the Gambia. Health Policy and Planning Advance, 1-11.

[29]. Shamsul Azhar S, A. N. (2012). Factors Influencing Childhood Immunization Defaulters in Sabah, Malaysia. THE INTERNATIONAL MEDICAL JOURNAL Malaysia.

[30]. World Health Organization (2009). Expanded Programme on Immunization: Routine Immunization Schedule. www.int.org 2009.

[31]. World Health Organization and United nations International Children Education Fund Global Vision and Strategy 2006-2015. (2005).

[32]. World Health Organization. (2005) World Immunization against diseases of public health importance. The cost effectiveness of immunization. www.int.org 2005

[33]. World Health Organization. (2008). Expanded Program on Immunization Training Manual. WHO/IVB

[34]. World Health organization. (2014) UnVacc Tools

[35]. Yihunie Lakew1, A. B. (2015). Factors influencing full immunization coverage among 12-23 months of age children in Ethiopia: evidence from the national demographic and health survey in 2011. BMC Public Health, 15:728.

[36]. Yoshito Kawkatsu, S. H. (2012). Individual, family and community level determinants of full vaccination coverage among children aged 12-23 months in western Kenya. Vaccine 30, 7588-7593.

[37]. Zimbabwe Demographic and Health Survey. (2010-2011). Key findings report. 